\title{
Research on Health Evaluation and Operation and Maintenance Decision of Intelligent Distribution Network Based on Health Index Theory
}

yang yang ( $\sim$ 365493675@qq.com )

China University of Petroleum

Research

Keywords: Health Index Theory, Intelligent Distribution Network, Health Degree Image

Posted Date: September 7th, 2021

DOl: https://doi.org/10.21203/rs.3.rs-779484/v1

License: (c) (1) This work is licensed under a Creative Commons Attribution 4.0 International License.

Read Full License 


\title{
Research on Health Evaluation and Operation and Maintenance Decision of Intelligent Distribution Network Based on Health Index Theory
}

Yang Yang 1

1 Shengli College, School of Mechanical and Control Engineering, China University of Petroleum, Dongying Shandong China, 257061.

E-mail: 000207@slcupc.edu.cn

\begin{abstract}
To solve the problems of dynamic state evaluation of large-scale distribution equipment and complex and changeable smart grid, and realize efficient planning, operation and maintenance. Based on the existing literature, this paper explores the distribution network health index theory and distribution network operation and maintenance strategy. The distribution network planning method based on health index is studied. Based on the existing power system research indexes, the suitable intelligent grid evaluation indexes are selected from the aspects of safety, economy, reliability and environmental protection, and an index system for comprehensive evaluation of smart distribution network health is selected. The critical characteristics of distribution network are classified, the indexes are established and the distribution network health grades are divided by analytic hierarchy process and entropy weight method. In this paper, the evaluation method is used to calculate the health index, classify the health degree and generate the health degree image for seven lines in a $110 \mathrm{kv}$ substation in oil field. According to the optimal operation and maintenance strategy of distribution network based on health index theory, it provides a reliable basis for distribution network planning, construction, operation and maintenance.

Keywords: Health Index Theory; Intelligent Distribution Network; Health Degree Image.
\end{abstract}




\section{Introduction}

With the increase of power load, access of distributed generation, popularization of electric vehicles, etc. smart power grid has gradually developed. Intelligent power distribution network is different from traditional power distribution network in safety, reliability, quality, economy and environmental protection.[1] The intelligent grid condition monitoring is not limited to the monitoring and managementing grid equipment, condition maintenance and life management, etc. but further developed into risk-based maintenance and continuously expanded to many fields such as safe operation, optimized dispatch, economical operation and service. The development of all intelligent grids cannot be separated from the understanding of existing equipment and network health. Based on traditional distribution network evaluation research, not only considering the equipment failure rate, but also considering the characteristics of intelligent distribution network.[2] This paper studies the influencing factors of intellectual distribution network health status, screens the evaluation indexes that can evaluate the health status of smart distribution network, and establishes the index system of distribution network health evaluation. At the same time, the operation and maintenance of distribution networks is an important measure to ensure the safe and stable operation of distribution network and the reliability of power supply.It is one of the most critical issues that enterprises engaged in power system work should pay attention to. Therefore, it is reasonable to analyze and put forward the operation and maintenance strategy of distribution network, which can not only ensure the reliability of power supply to the greatest extent, improve the maintenance efficiency, reduce the outage time, but also improve the economic benefits and system operation benefits of power grid enterprises. Literature [3] features are used in the data prediction by adopting the merge layer of the neural network. The experimental results show that the proposed MNMF model has better performance compared with the other methods in many evaluation indicators.. Literature [4] propose an improved DV-hop method after analysis of past research showed the need to significantly reduce the errors during the hop-distance conversion process and the relation between the estimated 
location and estimated distance. The improved method is based on the rigorous theoretical derivation to ensure correctness. Literature [5] proposes a data-driven modeling method based on deep neural network, which is used for vehicle calculation and prediction.

In recent years, the health index HI based on the real-time running status of equipment has been introduced into the power system.[6] The health index evaluation method was initially used in human medicine, and now it has developed in many areas. As a new method of distribution network health assessment, the distribution network health index quantifies the operation state of distribution network into a total value by processing and comprehensively calculating the key features that affect the health of distribution networks. In this way, the health status and fault warning of distribution network can be reflected more intuitively and clearly, and the overall operation and management level of power grid can be improved.

Health index theory was initially put forward by EA Company of Britain to solve many aging problems and better manage these equipment scientifically [7], and based on CBRM (Condition Based Risk Maintenance), the running status of distribution equipment was evaluated [8-9]. The system is based on the power operation database of European and American countries.It has been successfully applied in many power companies in Britain, as well as countries such as America, Canada and Australia. After that, Professor Ma Zhao put forward the concept of power equipment health index through joint research with British partners, trying to use the health index of power equipment to reflect the performance and life expectancy of power equipment [10], and British power grid company took the lead in using CBRM model [11] which can quantify equipment health index for distribution equipment maintenance; In 2003, scholar D. Hughes[12-14] first put forward the concept of power system health index, and set out to build a health index model and apply it to practice, which provided a research direction for power system health evaluation methods; In 2004, CBRM was used to the regulatory agencies in the UK, and the first edition of PAS 55 (Publicly Available Specification) [15] was released for asset management planning of the power industry; In 2006, D. Hughes further expanded the health index theory, which 
can evaluate the health status of power system more accurately. In the second year, the health index theory was further combined with the risk assessment system to quantify the risks, and the importance of risk classification was put forward [16]. At the same time, EA Company also introduced the second generation CBRM model, which further improved the health model of equipment and made the health assessment of distribution equipment more reliable. PAS 55 has gradually improved its system, and has become the most widely used and safest standard in power network health analysis in various countries. In 2014, it became the standard recognized by ISO 55000 [17]. In the following years, the United States, Australia, Britain and other countries also have more research results in the theoretical system of power system health, as shown in Table 1:

Table 1 Achievements Exhibition of Some Foreign Companies

\begin{tabular}{llllr}
\hline American Electric & Power & Australia Yuewang Company & \multicolumn{2}{l}{ Office of Natural Gas Power } \\
Research Institute & & & $\begin{array}{l}\text { Market, UK Industry } \\
\text { Regulatory Authority }\end{array}$ \\
\hline Establish a life cycle & Extended Risk Matrix Graph & Establishing & RIIO \\
management system for & Method for Semi-quantitative & Management & Mechanism \\
nuclear power system & Analysis & Based on Health Index (HIs) \\
structures and components & & & \\
\hline
\end{tabular}

In the literature [18], Professor Ju Ping first put forward the concept of power system health diagnosis and established a diagnosis model, analyzed and studied the health degree of the system through the change of the system running state, accurately located the cause of the fault, and then gave the fault prompt and early warning. Soon after, Professor Sun Hongbin supplemented the research, introduced the concepts of consultation and triage, and analyzed the evaluation system by using AHP and fuzzy theory, and established a set of reference models for measuring the health status of power grid [19]; In the literature [20], Ma Zhao and others began to systematically introduce the concept of health index theory, and analyzed and discussed the key characteristic quantities of distribution equipment and network, the calculation process of health index and the classification standard of health degree. Literature [21] characterizes the main key features affecting distribution equipment and distribution 
network by consulting data, analyzing actual situation and calculating data. Document [22] introduces the key features that have an impact on the line operation, and establishes a line risk assessment model based on PCA dimension reduction for line risk assessment. In the literature [23], the complex distribution network was diagnosed based on analytic hierarchy process (AHP), and the 10KV power network was taken as an example for verification. Literature [24] studies and analyzes the health and importance of distribution network operation, and gives a specific formula for calculating distribution network health index. Literature [25] will summarize the evaluation process of distribution network health index, and put forward the evaluation method based on group equipment and network. Literature [26] discusses the key characteristics of different distribution equipment in $10 \mathrm{kv}$ distribution network, and establishes a health model by using Logistic regression method for verification through 30 transformers as an example. Literature [27] studies the distribution network operation and maintenance, mainly including the importance, shortcomings and optimization methods of operation and maintenance emergency repair. Literature [28] is based on the business requirements of distribution network operation and maintenance, and studies the construction of distribution network operation and maintenance management platform.

This paper studies the distribution network health index theory and classification standard at present at home and abroad, and analyzes the optimal operation and maintenance strategy of power network. In this paper, the index evaluation system of distribution network is put forward, which considers many working conditions and development trends, such as bad working conditions of equipment, operating environment of equipment, safe operation of power grid, economy and service, etc. At the same time, the power factor, line voltage deviation and line loss rate of seven $110 \mathrm{kV}$ lines in an oilfield substation are selected as the characteristic quantities of health index division, and the key characteristic quantities are weighted by entropy method to complete the distribution network health degree classification and calculation. Finally, through the generated health image and health index analysis, the fault warning and push operation and maintenance strategy are prompted. 


\section{Determination of evaluation index of intelligent distribution network}

\subsection{Definition of Health Index}

Professor Ma Zhao put forward in "Research on the Theory and Application of Modern Distribution Network Health Index" [15] that the health index is to concretize the abstract power grid operation state through quantitative methods, and to measure and display the power grid operation state clearly and intuitively through specific numerical values. The health index can be obtained through logical analysis and mathematical operation based on the key characteristic quantities affecting the power grid operation. For the distribution network, the health index can comprehensively show the current running state of the network. The health status evaluation index of smart grid has a wide range of design and many factors to consider, so it is necessary to select the appropriate evaluation index according to the characteristics of smart grid.Oil field distribution network is a complex network system with real-time balance, discreteness and continuity, so it is difficult to describe the health of complete oil field distribution network from a single dimension. How to extract scientific and effective health evaluation indicators of smart grid from various key factors affecting distribution network and equipment is one of the research focuses of this paper. In this paper, the distribution network health index is comprehensively characterized from four dimensions: safety, reliability, economy and green on the basis of consulting domestic and foreign literature [15], combining with the statistical analysis of fault causes in actual operation of power system distribution network, and referring to the following regulations and standards: Guidelines for State-owned Distribution Network Maintenance and Operation Regulations of State Grid Corporation of China.

\subsection{Establishment of evaluation index}

In this paper, after consulting domestic and foreign literature [16], combining with the statistical analysis of fault causes in actual operation of power system distribution network, and referring to the following rules and standards: "Guidelines for Condition-based Maintenance of Distribution Network" and "Operation Rules of 
State Grid Corporation Distribution Network", it is decided to classify the key characteristics of distribution network health from four dimensions: safety, reliability, economy and environmental protection.

(1) Safety means that the distribution network should have the ability to resist certain risks and disturbances, and have the ability to deal with emergencies. Even if the distribution network is disturbed during operation, it can continue to maintain the power supply of the distribution network and keep the normal operation of the distribution network. Generally, load change rate, power factor and PV curve slope are the indexes to measure the safety of distribution network. Load change rate: the load change rate is too large, and if the available resources are insufficient, it will lead to abnormal voltage, which will affect the safety of equipment and distribution network. The load change rate is measured by the wallpaper of load change amount and peak value per unit time. Slope of PV curve: The greater the slope, the worse the voltage stability. Power Factor In order to reflect the utilization rate of AC power supply in the operation process of power system, the power factor is proposed, which is obtained by the ratio of active power to apparent power.

(2) Reliability refers to the ability of distribution network to continuously supply power to oilfield loads according to specified quality standards and required power, that is, the ability of "guaranteeing quality and quantity"-ensuring the quality and continuity of power supply of distribution network. Usually, line voltage deviation, fault recovery time and load margin are used. Line voltage deviation means that the deviation degree between the actual voltage of each line and the standard voltage of the line becomes the line voltage deviation. Load margin: it measures whether the current state of the power grid is stable. The smaller the index value, the smaller the load margin of the system, reflecting the greater the load pressure of the system. Failure recovery time refers to the time from failure to recovery to current state.

(3) Economy refers to the operation economy of distribution network under typical load conditions, which reflects the loss situation, operation and maintenance cost and overall system operation efficiency of distribution network in oilfield. The measurement indicators are line loss rate, network loss and operation and maintenance 
cost ratio. The so-called line loss rate refers to the percentage of the power lost in the process of line transmission to the total power provided by the power plant. According to different calculation methods of line loss, it can be divided into statistical line loss rate and theoretical line loss rate . (4) Environmental protection index refers to the access of clean energy and the popularization of electric vehicles, which not only alleviates the dependence on fossil energy, but also greatly reduces the emission of greenhouse gases. In this paper, the permeability of distributed energy is selected as the environmental protection index. The higher the permeability, the higher the proportion of clean energy in the whole system, the better the environmental protection.

Table 2 The classification table of key characteristic quantities of smart grid:

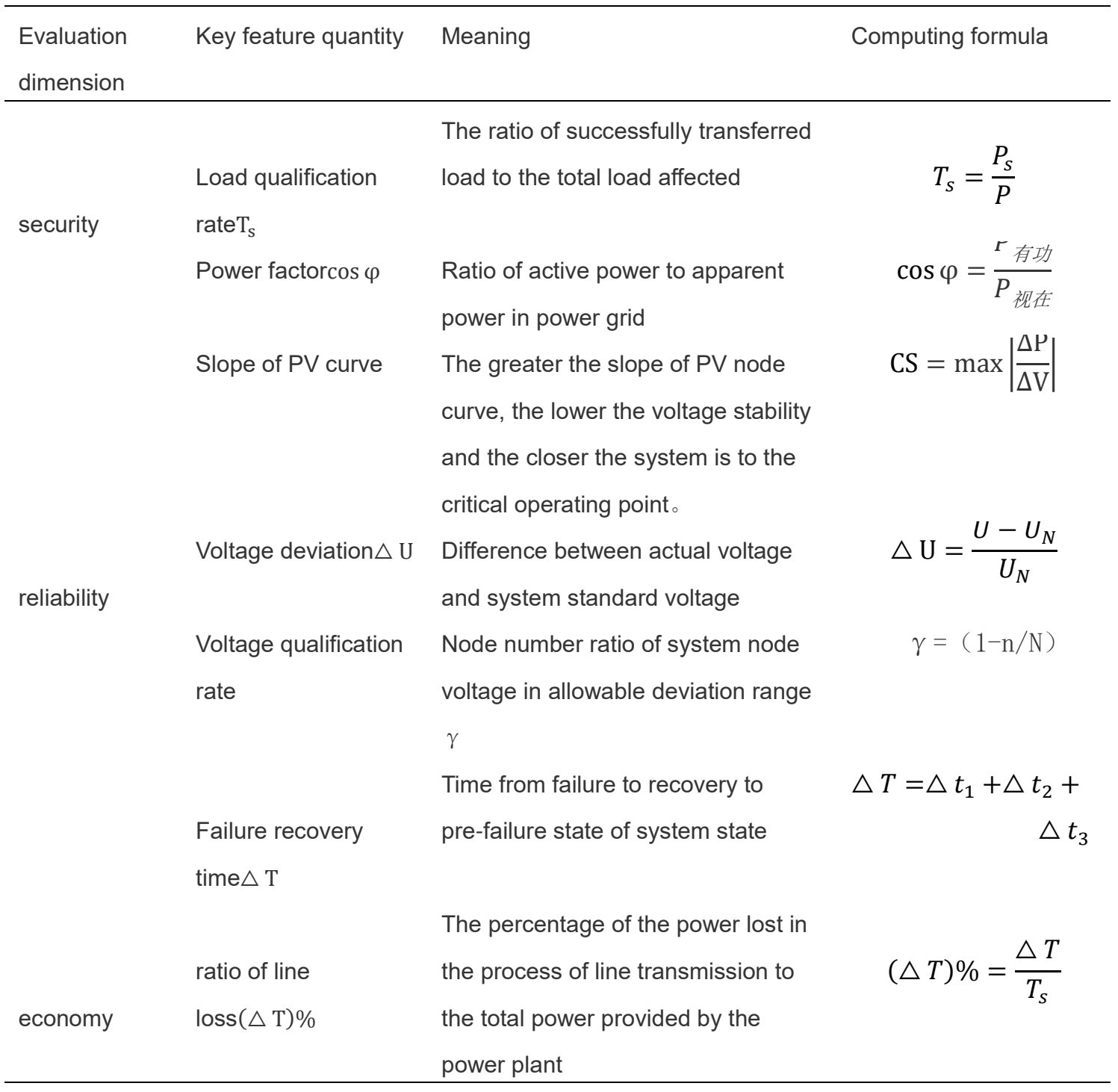




\begin{tabular}{|c|c|c|c|}
\hline & $\begin{array}{l}\text { Proportion of } \\
\text { operation and } \\
\text { maintenance } \\
\text { expensesC }_{\mathrm{s} 2}\end{array}$ & $\begin{array}{l}\text { The proportion of network operation } \\
\text { and maintenance expenses to total } \\
\text { electricity sales }\end{array}$ & $\mathrm{C}_{\mathrm{s} 2}=\frac{\mathrm{C}_{\mathrm{M}}}{\mathrm{W}_{\mathrm{s}} \mathrm{P}}$ \\
\hline $\begin{array}{l}\text { Environmental } \\
\text { protection }\end{array}$ & $\begin{array}{l}\text { Penetration rate of } \\
\text { distributed } \\
\text { energy } \lambda\end{array}$ & $\begin{array}{l}\text { The ratio of } \mathrm{Pi} \text { distributed } \\
\text { generation to the maximum load } \\
\text { power of power gridPLmax }\end{array}$ & $\lambda_{\mathrm{DG}}=\sum \frac{\mathrm{Pi}}{\mathrm{PLmax}}$ \\
\hline
\end{tabular}

\section{3 smart grid health classification}

According to the evaluation index determined in Section 2, the running state of smart grid is reasonably divided, and the criteria for dividing the value range of health degree according to the existing literature and Guidelines for State Evaluation of Distribution Equipment are summarized, and the distribution network is divided into five states, namely health, sub-health, general defects, serious defects and urgent defects, as shown in the following table3.

Table 3 Classification of Health Degree of Oil Field Distribution Network

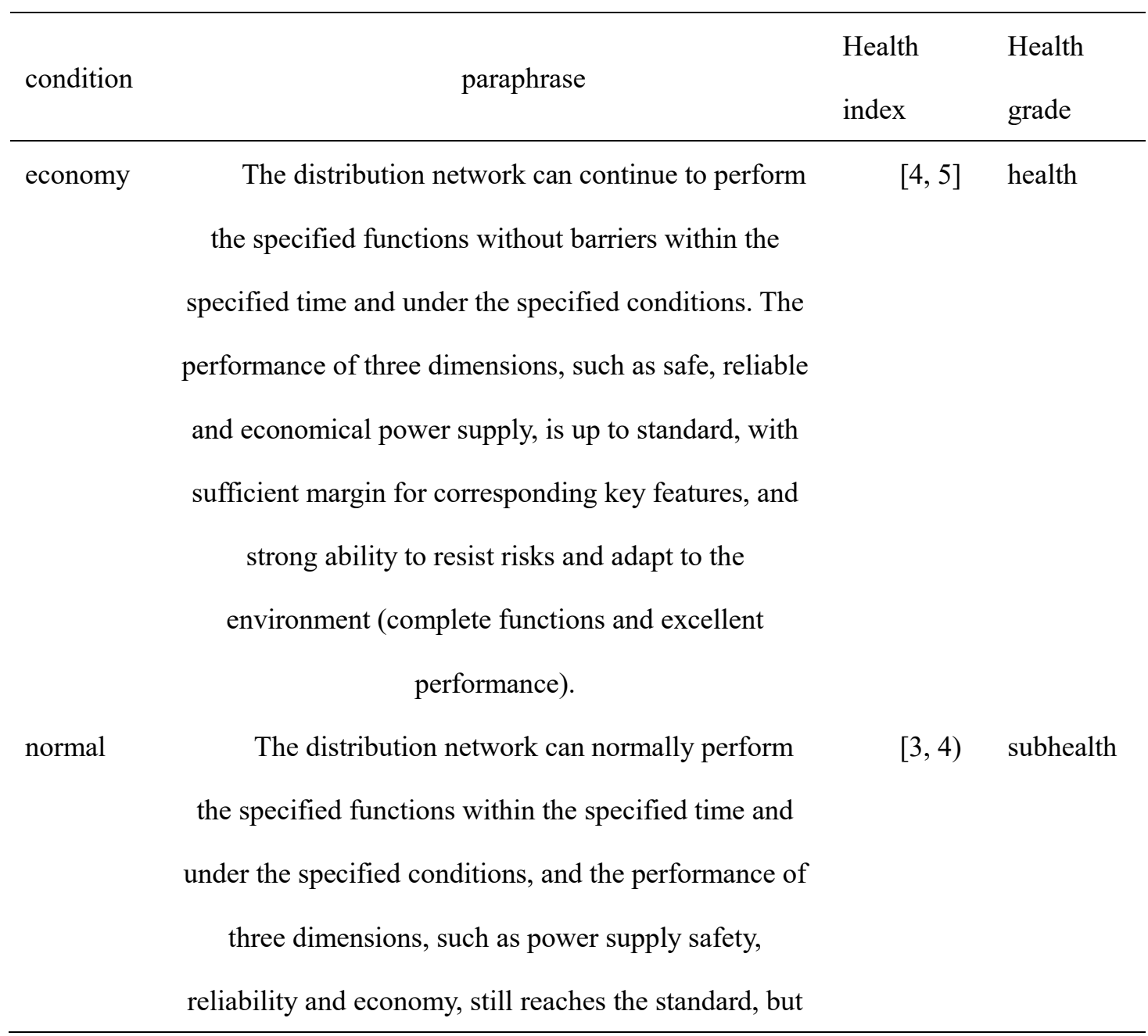


some key characteristic quantities are close to the

standard limits, and the ability to resist risks and adapt

to the environment declines (complete functions,

performance decline).

fragile The distribution network can normally perform

$[2,3) \quad$ General

the specified functions within the specified time and

defects

under the specified conditions, but the performance of

three dimensions, such as power supply safety,

reliability and economy, has been partially degraded,

and the corresponding characteristic indexes are

over-limited, but the comprehensive influence degree is

small, and the ability to resist risks and adapt to the

environment has slight defects (complete functions and

slight defects in performance).

worsen

The distribution network can normally perform the specified functions within the specified time and

$[1,2)$ major

defects

under the specified conditions, but the performance of power supply safety and reliability has been seriously degraded in part, and the corresponding characteristic indicators are over-limited, resulting in a large comprehensive impact, and obvious defects in risk resistance and environmental adaptability (complete functions and obvious defects in performance).

extreme The distribution network has been unable to

$[0,1) \quad$ Critical perform the specified functions normally, and the defect performance of power supply security and reliability has been seriously degraded, resulting in serious consequences. 


\section{Smart grid HI calculation}

\subsection{Selection of key feature quantities}

In the process of power system operation, the power factor of distribution network can effectively reflect the power grid operation. Generally speaking, when the distribution network is in normal operation, the power factor is in a dynamic equilibrium state. When the power factor drops to a certain value, it will lead to excessive reactive power, which will lead to a decrease in the power factor of the whole power system and affect the operation of the whole power system. During the operation of the distribution network, the load and the output of the generator set change in real time, which also leads to the reactive power change of the distribution network and the voltage deviation. If the voltage deviation is too large or too small, it will seriously affect the operation performance of electrical equipment, reduce the use efficiency of equipment, and at the same time, it will greatly reduce the transmission limit of transmission lines, resulting in the unstable operating frequency of the system, eventually leading to the collapse of the system operation, which greatly affects the reliability of the system operation. According to the survey data, as of 2019, China's total power generation was 7,503.43 billion kilowatt hours, up by $4.5 \%$ year-on-year. Although the power generation is growing rapidly, the national line loss rate in China in 2019 was as high as $5.9 \%$, which was about $2 \%$ higher than that in developed countries, so the power loss in transmission is still serious [27]. At present, China vigorously advocates energy-saving policies, and State Grid Corporation has also formulated and promulgated the Regulations on Power Loss Management in Power Network and Guidelines for Calculation of Power Loss in Power Network [28] and other standards to limit the increase of line loss rate. As an important part of the power system, the distribution network should also reduce the loss in the transmission process, so as to improve the economy of power grid operation. To sum up, in this paper, the three data of power factor, line voltage and line loss rate are used as the key characteristics of distribution network to calculate the health index. 


\subsection{Calculation of Key Feature Weight}

In this paper, entropy weight method is adopted to obtain the weight, which is an objective weighting method. According to the definition of entropy, for a certain index, its entropy value can be used to judge the discrete degree of the index. The greater the discrete degree, the greater the impact of the index on the overall comprehensive evaluation. Therefore, entropy weight method can be used to calculate the weight of each index, which provides a basis for multi-index comprehensive evaluation. In this paper, the five-day health index of oilfield distribution network is evaluated. There are three indexes in the system, so the steps of comprehensive evaluation by entropy method are as follows:

(1) Normalize the key feature quantities Among them,

The power factor and line voltage are positive indicators (i.e. the greater the value, the smaller the impact on the health of distribution network), so the calculation method is shown in Formula (3-1):

$$
y_{i j}=\frac{x_{i j}-x_{\max }}{x_{\max }-x_{\min }}
$$

The line loss rate is an inverse index (that is, the greater the value, the greater the influence on the health of distribution network), so the calculation method should adopt formula (3-2):

$$
y_{i j}=\frac{x_{\max }-x_{i j}}{x_{\max }-x_{\min }}
$$

Where: $y_{i j}$ is the normalized value; $x_{i j}$ is the value without normalization; $x_{\max }$ is the maximum value in the current range; $x_{\min }$ is the minimum value in the current range.

Through the above calculation, all the key feature quantities are transformed into unitless relative values, and the values are within $[0,1]$. So as to facilitate the subsequent calculation of weights.

(2) Calculate entropy and redundancy:

The entropy value is calculated by formula (3-3):

$$
E_{j}=-\frac{1}{\ln n} \sum_{i=1}^{n} p_{i j} \ln p_{i j}
$$


Where: $E_{j}$ represents the entropy value of the key characteristic quantity; $p_{i j}$ represents the proportion of normalized key features in the whole range.

Entropy is a measure with uncertainty. The greater the amount of information, the smaller the uncertainty and entropy. The smaller the amount of information, the greater the uncertainty and entropy. According to the characteristics of entropy, entropy is used to judge the discrete degree of an index. The greater the discrete degree of an index, the greater the impact of the index on comprehensive evaluation. Therefore, according to the variation degree of each index, the weight of each index can be calculated by using the tool of information entropy, which provides a basis for multi-index comprehensive evaluation.

(3) Calculate the difference coefficient and weight of key feature quantities:

The difference coefficient and weight of key feature quantities can be calculated by formulas (3-4) and (3-5):

$$
\begin{array}{r}
d_{j}=1-e_{j} \\
w_{j}=\frac{d_{j}}{\sum_{j=1}^{m} d_{j}}
\end{array}
$$

Where: $d_{j}$ is the difference coefficient $0 ; w_{j}$ is the weight of the first index.The health degree of distribution network is calculated from three dimensions: safety, reliability and economy, in which the safety dimension is measured by power factor; The reliability dimension is measured by line voltage. Economic dimension is measured by line loss rate.

\subsection{Calculation of Health Index}

The real-time health index of oilfield distribution network can be obtained by normalizing, grading and multiplying the data of three key feature quantities at any time by the index weight value and then adding them together, so as to obtain the final evaluated health degree of oilfield distribution network. In this paper, the calculation formula [18] used to describe the health index of power system distribution network running state at any time is as shown in Formula (3-6):

$$
H_{(t)}=\frac{\sqrt{\sum_{j=1}^{M}\left(w_{j}(t) G_{j}(t)\right)^{2}}}{\sqrt{\sum_{j=1}^{M} w_{j}^{2}(t)}}
$$


Where: it is the weight value determined by different key feature quantities, and the influence degree of each key feature quantity on health degree can be seen by setting the weight; The health grades determined for different key feature quantities are obtained by dividing the key feature quantities into health grades;

\section{Operation and maintenance strategy}

According to the three key characteristic quantities of power factor, line voltage deviation and line loss rate selected in this paper, there are two main methods to improve the power factor of distribution network:

(1) improve the natural power factor: 1) try to make the distribution network run at full load; 2) Reasonably distribute the distribution network layout and improve the actual operation of the distribution network; 3 ) Adopt electromagnetic switch with no pressure operation.

(2) Reactive power compensation: 1) adopt synchronous compensator to make distribution network work in overexcitation state; 2) Capacitors are connected in parallel to generate capacitive current to compensate inductive current generated by distribution network for reactive power compensation.

Reducing line voltage deviation can be solved by reducing line impedance and boosting voltage:

(1) reduce the line impedance 1) replace overhead lines with cables; 2) In areas not suitable for overhead lines, the line reactance is compensated by series capacitors to reduce the line impedance.

(2) boosting 1) Install phase-shifting capacitors to stabilize the voltage during distribution network operation; 2) Adopt automatic adjustment system to adjust the voltage in real time. To reduce the line loss rate, the following points can be considered from the aspects of power grid capacity, reasonable distribution, balance of three-phase load and equipment management: (1) PRTV anti-pollution flashover material is sprayed on the insulator surface, which prevents the direct contact between dirt and insulator and indirectly reduces the line loss rate; (2) Reduce the line loss rate by adjusting and increasing the climb; 
(3) Regularly or irregularly inspect the distribution network equipment and replace the old equipment in time.

To reduce the line loss rate, the following points can be considered from the aspects of power grid capacity, reasonable distribution, balance of three-phase load and equipment management: (1) PRTV anti-pollution flashover material is sprayed on the insulator surface, which prevents the direct contact between dirt and insulator and indirectly reduces the line loss rate; (2) Reduce the line loss rate by adjusting and increasing the climb; (3) Regularly or irregularly inspect the distribution network equipment and replace the old equipment in time. Figure 1 shows the push process of operation and maintenance countermeasures. In an example, in order to better compare the changes of line loss health in one day, the line health portraits are displayed on the big data platform every day. It is stipulated that when the average health index of a certain line on that day is lower than 3 , the big data platform will issue a warning, that is, the health of a specific line shows general defects/serious defects/critical defects. Then analyze the reasons of health deterioration according to the health index, and push the line loss improvement strategy according to the deterioration factors: (1) when the average power factor is lower than 0.7 , push the "reactive power compensation" strategy; (2) When the average line voltage is lower than $85 \%$, push the "boost" strategy; (3) When the power factor is higher than 0.7 and the line voltage is higher than $85 \%$, it means that the line itself is trouble-free, and the deterioration of the line loss may be due to the problems in the readings of the outlet power quantity and the inlet power quantity. "Data measurement is wrong, and the line is healthy". 


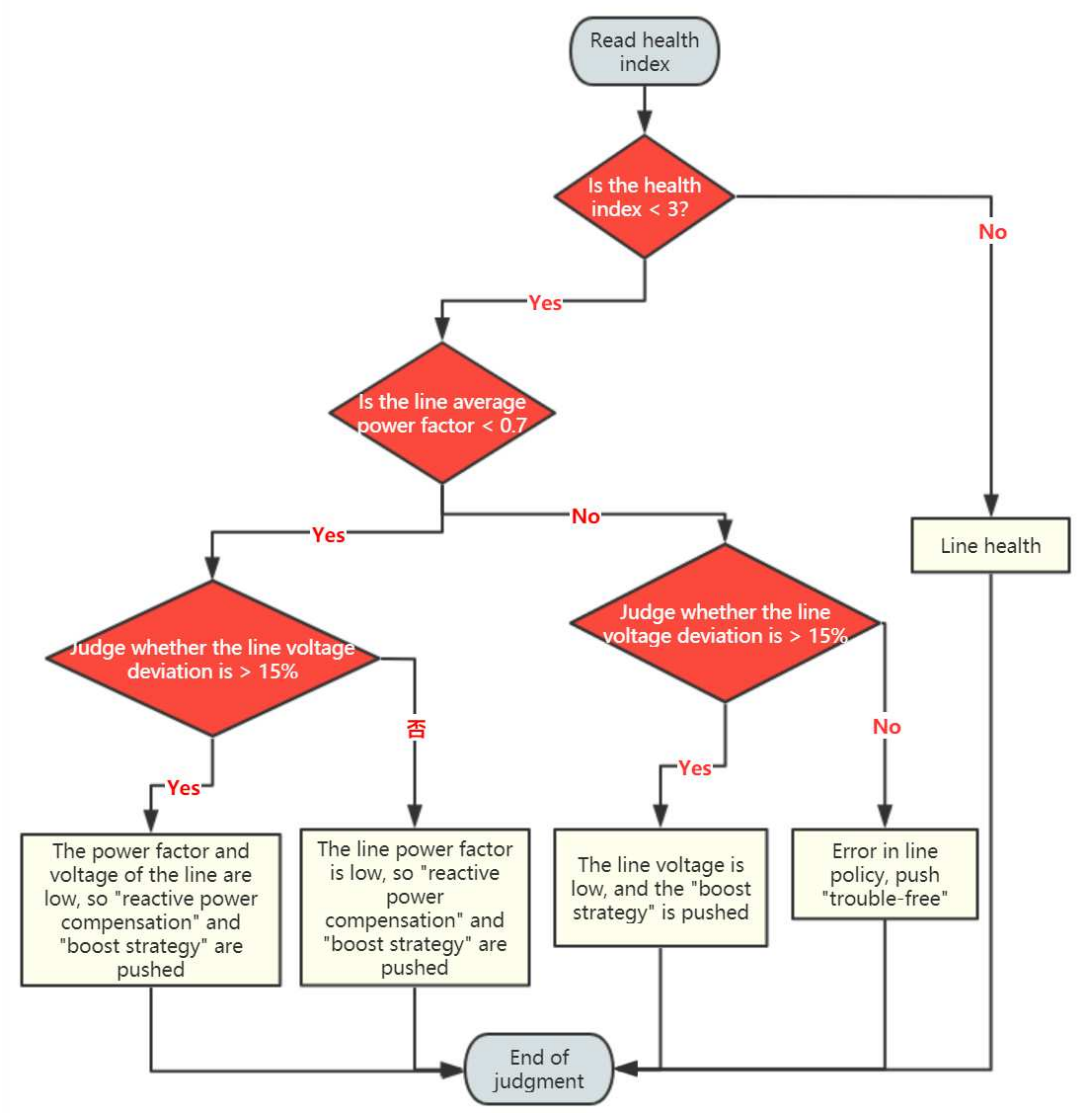

Fig1 The push process of operation and maintenance countermeasures

\section{Examples of Health Division}

\subsection{Calculation of Health Weight Index}

In this paper, the power factor, voltage deviation and line loss rate of 7 lines of $110 \mathrm{KV}$ distribution network in an oilfield are taken as key characteristic quantities to calculate the network health index. In an example, the health degree portraits of 7 lines including $\mathrm{A} \sim \mathrm{G}$ in 4 days, 4.24 days, 4.30 days, 5.2 days, 5.27 days and 5.31 days are made respectively. In the figure, the abscissa represents time with an interval of 1 hour, and the ordinate represents the line. Firstly, the health grade $G$ is determined according to the key feature quantity, and then the final health grade $\mathrm{H}$ is obtained by weighting. The line loss rate, line voltage deviation percentage and power factor weight are $0.6,0.15$ and 0.25 respectively.

(1) voltage deviation calculation process shows: Taking the data of line A at 7:00 on April 24, 2020 as an example, it can be known from the data that the voltage level of the distribution network system is $110 \mathrm{KV}$, and the actual operating voltage of the 
line is $105 \mathrm{KV}$, which can be obtained from Formula 5-1:

$$
\triangle U=\frac{105-110}{110} \times 100 \%=-4.5 \%
$$

That is, the voltage deviation of line a in this period is $-4.5 \%$, and the voltage deviations of other lines are also obtained by the above method. At present, when the distribution network of China's power system operates normally, the voltage deviation value of most lines does not exceed $10 \%$, so the health level of voltage deviation is divided according to this standard as shown in Table 4:

Table 4 Health Classification of Line Voltage Deviation

\begin{tabular}{cc}
\hline Line voltage deviation & Health grade \\
\hline$[0,10 \%)$ & 5 \\
{$[8 \%, 15 \%)$} & 4 \\
{$[15 \%, 20 \%)$} & 3 \\
{$[20 \%, 30 \%)$} & 2 \\
{$[30 \%, \infty)$} & 1 \\
\hline
\end{tabular}

(3) Show the calculation process of line loss rate: Taking the data of line A at 7: 00 on April 24, 2020 as an example, it can be known from the data that power supply and sales of the distribution network in this period are $384 \mathrm{~kW}$ and $345.5 \mathrm{~kW}$, which can be obtained by formulas $5-2$ and 5-3:

$$
\begin{aligned}
\triangle T=384-345.5 & =38.5 \mathrm{KV} \\
& (\triangle T) \%=\frac{38.5}{384}=10 \%
\end{aligned}
$$

That is, the line loss rate of line a in this period is $10 \%$, and the rest line loss rates are also obtained by the above method. When the distribution network of China's power system operates normally, the line loss rate of most lines does not exceed $10 \%$, so the health level of line loss rate is divided according to this standard as shown in

\begin{tabular}{|c|c|}
\hline Ratio of line loss & Health grade \\
\hline$[0,10 \%)$ & 5 \\
\hline$[10 \%, 20 \%)$ & 4 \\
\hline$[20 \%, 30 \%)$ & 3 \\
\hline$[30 \%, 50 \%)$ & 2 \\
\hline
\end{tabular}
Table 5:

Table 5 Health Classification of Line Loss Rate 
$[50 \%, \infty)$ 1

(3) The power factor is directly obtained from the system operation measurement, and is not calculated in this paper. When the distribution network is in normal operation, the power factor should not be lower than 0.9 , so the health level of power factor is divided according to this standard as shown in Table 6:

Table 6 Classification of Power Factor Health Level

\begin{tabular}{cc}
\hline Power factor & Health grade \\
\hline$\left[\begin{array}{ll}0.85, & 1\end{array}\right)$ & 5 \\
{$\left[\begin{array}{ll}0.7, & 0.85\end{array}\right)$} & 4 \\
{$\left[\begin{array}{ll}0.6 & 0.7\end{array}\right)$} & 3 \\
{$\left[\begin{array}{ll}0.5, & 0.6\end{array}\right)$} & 2 \\
{$\left[\begin{array}{ll}0, & 0.5\end{array}\right)$} & 1 \\
\hline
\end{tabular}

The value of health degree in the above three tables is $(0,5)$, and different values correspond to different states. The larger the value, the better the health state. They respectively correspond to five grades: health $(4,5)$, sub-health $(3,4)$, general defect $(2,3)$, serious defect $(1,2)$ and critical defect $(0,1)$, which are also divided according to these five grades when depicting the health degree portrait of oil field distribution network. The five colors represent the health status of five oilfield distribution networks, for example, green indicates that the health level is at $(4,5)$, that is, health status, and red indicates that the health level is at $(1,2)$, that is, serious defect status. When the power factor is 0.781 , the line voltage deviation is $4.5 \%$, the corresponding health level is 5 , the line loss rate is $10 \%$, and after the corresponding health level is 5 , calculate the comprehensive health index of line 7 by formula $5-4$ :

$$
H_{(\mathrm{t})}=\frac{\sqrt{3^{2}+0.6^{2}+0.75^{2}}}{\sqrt{0.6^{2}+0.15^{2}+0.25^{2}}} \approx 4.72
$$

It can be obtained that the health index at line a 7 is about 4.72 , which corresponds to the health level.

\subsection{Health portrait results and examples of operation and maintenance} strategies

(1) health portrait results and operation and maintenance strategy on April 24th, 2020 


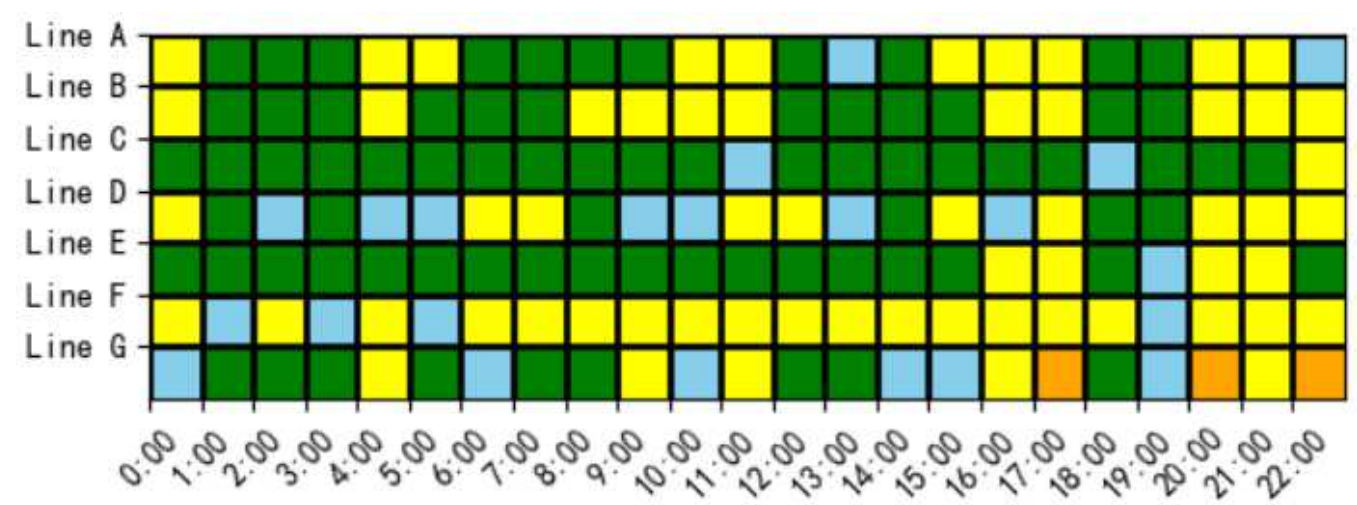

Fig.2 Image of oilfield health on April 24th

It can be seen from the health portrait in Figure 2 that Line $\mathrm{A}$ is in a healthy state for most of the day, and is in a general defect state and sub-health state for a small part of the day; B line is in a healthy state for most of the day, and a small part of the day is in a general defect state; $\mathrm{C}$ line is in a healthy state for most of the day, and it is in a general defect state and sub-health state for a few times; D line is in a healthy state and a sub-healthy state for most of the day, and a small part of the time is in a general defect state; E-line is in a healthy state for most of the day, and is in a general defect state and sub-health state for a small part of the day; F line is in general defect and sub-health state for most of the day; G-line is in a healthy and sub-healthy state in most time periods of the day, and in a few time periods it is in a state of general defects and serious defects. The health indexes of 7 lines are: Line A: 3.47475207 ; B: 3.47735188; C line: 4.41313853; D line: 3.25823759; E line: 4.0762806; Line f: 2.59389303; G line: 3.43516711. According to the classification of health degree, it is concluded that line $\mathrm{f}$ is in a general defect state on that day, and then the causes of defects are analyzed according to the program, and it is concluded that the average power factor of line $\mathrm{f}$ is higher than 0.7 , the voltage deviation is less than $15 \%$, and the push operation and maintenance strategy is: "data measurement is wrong, the line is healthy".

(3) Health degree portrait results and operation and maintenance strategies on May 2, 2020 


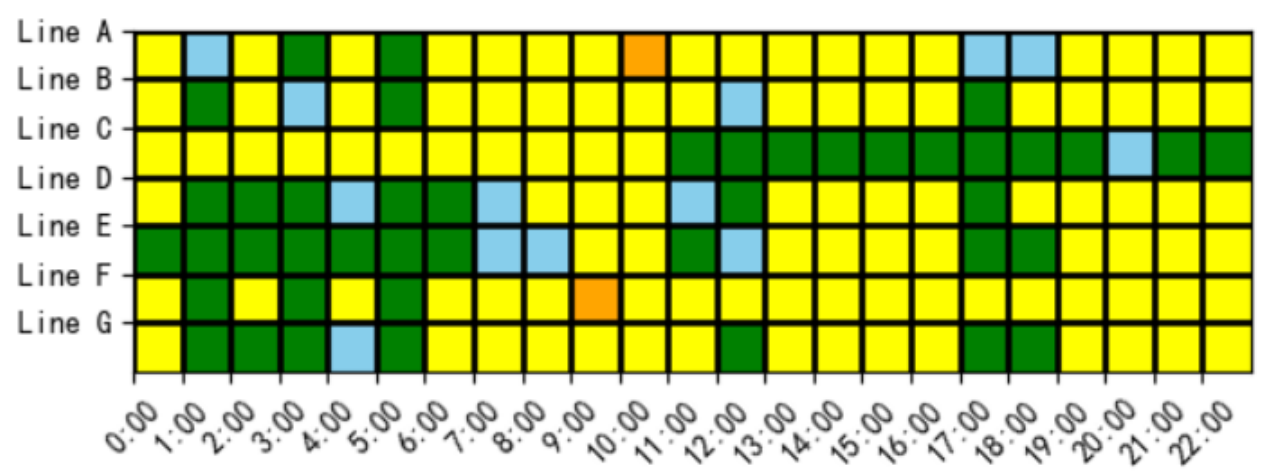

Fig. 3 health degree portrait of oil field on May 2

It can be seen from figure 3 health image that line a is in general defect state in most time periods of the day, and in sub-health state or general defect state or serious defect state in a very small time period; B line is in a general defect state for most of the day, and is in a sub-health state or healthy state for a very small part of the day; C-line, D-line and E-line are in a healthy or sub-healthy state in some time periods of the day, and the rest time periods are in a general defect state; F-line is in a general defect state in most time periods of the day, and a few time periods are in a healthy state, a sub-healthy state and a serious defect state; G-line is in a general defect state in most time periods of the day, while a few time periods are in a healthy and sub-healthy state. The average health index of the 7-day line is: Line A: 2.53806134; B line 2.6222447; C line: 3.52801937; D line: 3.34940636; E line: 3.23770822; Line f: 2.71030898; G line: 3.05460374. According to the classification of health degree, it is concluded that the A, B and F lines belong to general defect state on that day, and then the defect reasons are analyzed according to the program, and it is concluded that the power factor of the A, B and F lines is higher than 0.7 and the line voltage is higher than $85 \%$ on that day, so it is judged that the lines themselves are trouble-free, and the deterioration of line loss may be due to the problems of the readings of outlet power and inlet power, and "wrong data measurement, line health" is pushed.

(4) Results of health degree portrait on May 27, 2020 and operation and maintenance strategy 


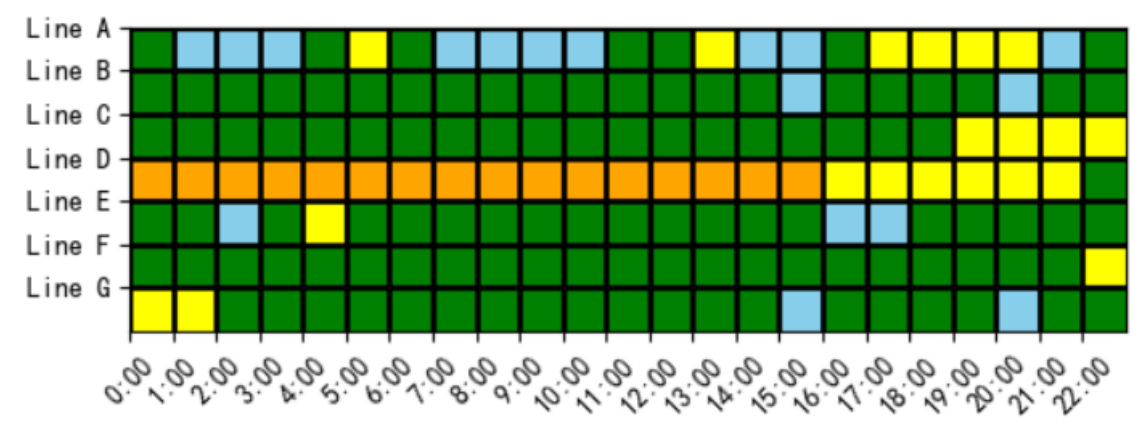

Fig.4 Health degree portrait of oil field on May 27

It can be seen from Figure 4 Health Image that Line A, Line E and Line G are in a healthy state or sub-healthy state for most of the day, and are in a general defect state for a very small time; B line has been in a healthy or sub-healthy state on the same day; C-line and F-line are in a healthy state for the most part of the day and in a general defect state for the least part of the day. D-line is in general defect state or serious defect state in most time periods of the day, and is in healthy state in a very small time period. The average health index of the 7-day line is: Line A: 3.34918725; B line 4.54276541; C line: 4.201211785; D line: 1.8757232; E line: 4.18757232; Line f: 4.33537418; G line: 4.05350089. The D-line is in a serious defect state on that day, and then the defect reason is analyzed according to the program, and it is concluded that the power factor of D-line is lower than 0.7 on that day, so the "reactive power compensation" strategy is pushed.

(4) Results of health portrait on May 31, 2020 and operation and maintenance strategy

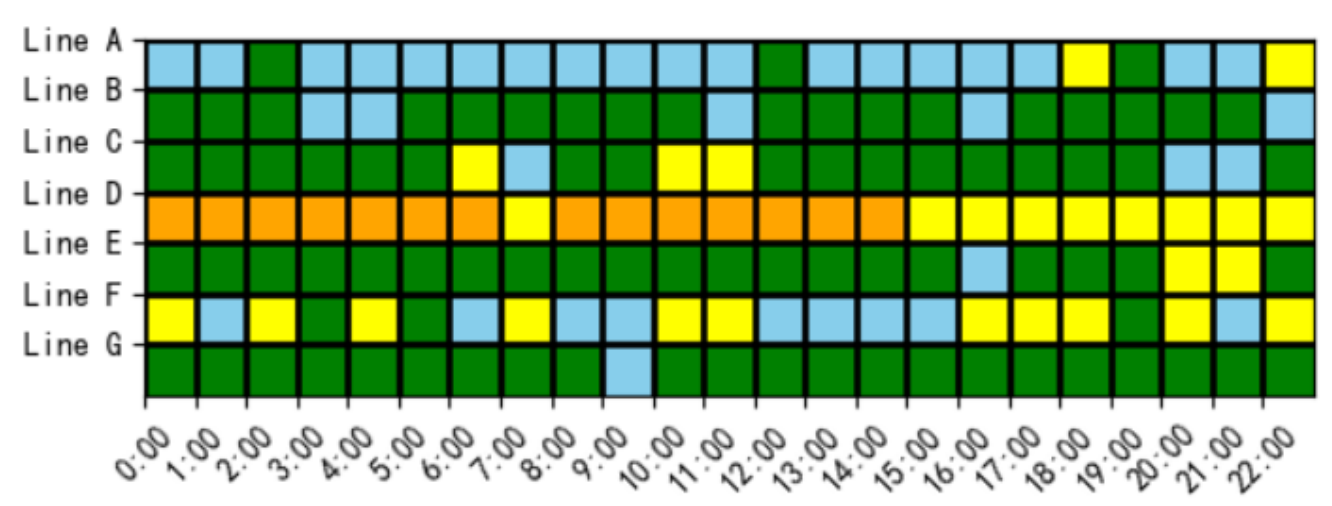

Fig. 5 health degree portrait of oil field on May 31

It can be seen from the health image in Figure 5 that the A-line, C-line and F-line are in a healthy state or sub-healthy state in most time periods of the day, and are in a 
general defect state in a very small time period; B-line and G-line were in a healthy or sub-healthy state all the time. D-line was in a general defect state on that day. The running results show that the average health index of the 7-day line is: Line A: 3.323441330; B line 4.27915817; C line: 4.38023766; D line: 1.88674324; E line: 4.02822595; Line f: 3.2207806; G line: 4.41277291. According to the classification of health level, it is concluded that the D line is in a serious defect state on that day, and then the defect reason is analyzed according to the program, and it is concluded that the line voltage deviation of the D line on that day is more than $15 \%$, so the "boost" strategy is pushed.

\section{Conclusion}

In this paper, the health index method is used to discuss the generation of distribution network health image and the operation and maintenance countermeasures, and the main conclusions are as follows: (1) Health degree evaluation method is one of the important methods for evaluating the health degree of oilfield distribution network, and the health degree index is obtained by weighting characteristic key quantities. (2) In the selection of key feature quantities, the line loss rate, power factor and line voltage are selected as the key feature quantities with three indexes of safety, economy and reliability as the standard; (3) Entropy weight method can be used to calculate the weight of key feature quantities. (4) First, determine the health grade $\mathrm{G}$ according to the key characteristic factors, and then obtain the final health grade $\mathrm{H}$ determined by all factors by weighting. In addition, different health grades are divided into colors, and finally, a health portrait of a certain line at different times and days can be generated.

\section{References}

[1] Liu weihuan. outage time model of distribution network maintenance and its application in reliability evaluation [D]. Chongqing university.

[2] Chen Tong. Health Analysis of Distribution Network Equipment Based on Big Data [J]. Communication Power Supply Technology, 2018,35 (11) 
[3] Hongju Cheng, Zhe Xie, Leihuo Wu, Zhiyong Yu \& Ruixing Li.Data prediction model in wireless sensor networks based on bidirectional LSTM[J].EURASIP Journal on Wireless Communications and Networking volume 2019.

[4] Xiaoyong Yan, Lijuan Sun, Jian Zhou and Aiguo Song.DV-hop localisation algorithm based on optimal weighted least square in irregular areas[J].Electronics Letters, 2019.

[5] Pan Yongjun; Nie Xiaobo; Li Zhixiong; Gu Shuitao.Data-driven vehicle modeling of longitudinal dynamics based on a multibody model and deep neural networks[J].MEASUREMENT.2021.109541.

[6] Guo huacheng. research on distribution network maintenance plan and decision optimization method based on health index [D]. north China electric power university (Beijing), 2018.

[7] Hughes D, Dennis G, Walker J, etal. Condition Based Risk Management (CBRM) - Enabling Asset Condition Information to be Central to Corporate Decision Making[J]. Engineering Asset Management, 2008:1212-1217.

[8] D.Hughes, Tracypears condition based risk management(CBRM)-a process to link engineering knowledge and practical experience to investment planningan update[C]//19st CIRED, 2007.

[9] D. Hughes. Condition based risk management(CBRM)- enabling asset condition information to be central to corporate decision making[C]//18st CIRED, 2005.

[10] Zhou Limei, Ma Zhao, Sheng Wanxing. The latest research progress of modern distribution network health index theory $[\mathrm{J}]$. Power Supply and Consumption, 2016,33 (01): 3-7+13.

[11] Li Yiqiang. Preliminary Study on Health Index of Power System Distribution Network [D]. North China Electric Power University, 2016.

[12] Hughes D, Pears T, Tian Y. Linking engineering knowledge and practical experience to investment planning by means of condition based risk management [C] . 
Condition Monitoring and Diagnosis, CMD 2008. International Conference on IEEE, 2008: 539-542.

[13] Hughes D, Pschierer-Barnfather P, Pears T. Condition based risk management (CBRM): bringing excellence in asset management into the boardroom[C]. Electricity Distribution-Part 1， 2009. CIRED 2009. 20th International Conference and Exhibition on. IET, 2009: 1-4.

[14] Hughes D, Barnfather P. Building risk based investment programmes[J]. 21st CIRED， 2011 .

[15] Ma Zhao, Zhou limei, sheng wanxing. analysis and comparison of international asset management standards ISO 55000 and PAS 55 [j]. power supply and utilization, 2015,32 (6): 48-53.

[16] Wang Rong. Research on Distribution Network Condition Evaluation Method Based on Equipment Health Index [D]. Xi 'an University of Technology, 2019.

[17] Ju Ping, Zuo Yingfei, Wen Fushuan, Wei Zhinong, Ma Hongzhong. Power System Health Diagnosis [J]. Electric Power Automation Equipment, 2004(06):22-25.

[18] Sun Hongbin, Hu Jiangyi, Liu Yingshang, Zhang Boming, Wu Wenchuan. Development of Dispatching and Control Center Function-Power Grid Real-time Security Early Warning System [J]. Power System Automation, 2004(15):1-6+14.

[19] Wang Dongsheng. Research on line loss calculation mismatch method and improvement strategy of Jinqiao Power Supply Branch [D]. North China Electric Power University (Beijing)

[20] Ma Zhao, Zhou Limei, Yuan Haiwen, Shi Shenxing, Liu Yingyi, Shang Yuwei, Sheng Wanxing, Su Jian. Research on modern distribution network health index theory and its application [J]. Proceedings of the CSEE, 2019,39 (01): $130-140+328$

[21] Sun Lingjie. Selection of key features based on distribution transformer health index [J]. Electronic World, 2020(09):77-78.

[22] Hu Zhipeng, Liu Jian, Zhang Bo, Wang Meirong, Kuang Shi, Yang Xuerui. Risk assessment of transmission line operation environment based on key risk 
characteristics [J]. Power System Automation, 2017,41 (18): 160-166.

[23] Shu, Tan Xijing, He Zhengyou, Zang Tianlei, Zhao Jing. Research on Health Diagnosis of Complex Distribution Network Based on Analytic Hierarchy Process [J]. Power System Protection and Control, 2013,41 (13): 7-13.

[24] Lin Yuan. Distribution network route evaluation based on health and importance [J]. Power Big Data, 2020,23 (08): 63-70.

[25] Huang Chang, Wang Chang, Yanghong Tan, Dong Xuzhu, Wu Zhengrong, Chen Chun. Distribution network operation risk assessment method based on health and importance [J]. Electric Power Automation Equipment, 2016,36 (06): 136-141.

[26] ma Zhao, Zhao mingxin, Zhou limei, Zhang Wei, Liu Wei, su Jian, sheng wanxing, yuan haiwen, Shi shenxing, Colin. an evaluation method of distribution network health index [P]. Beijing: CN105512448A, April 20, 2016. Guo huacheng. research on distribution network maintenance plan and decision optimization method based on health index [D]. north China electric power university (Beijing), 2018.

[27] Chen qingmei. research on optimization management of distribution network operation and repair [J]. business story, 2018(10):89.

[28] Shi Chang Kai, Zhang Bo, Sheng Wanxing, Zhou Meng, Eva, Li Yuling. Discussion on the functional architecture of distribution network operation and maintenance management platform [J]. Power Grid Technology, 2016,40 (07): 2206-2211.

\section{Methods/Experimental}

This paper studies the distribution network health index theory and classification standard at present at home and abroad, and analyzes the optimal operation and maintenance strategy of power network. In this paper, the index evaluation system of distribution network is put forward, which considers many working conditions and development trends, such as bad working conditions of equipment, operating environment of equipment, safe operation of power grid, economy and service, etc. At the same time, the power factor, line voltage deviation and line loss rate of seven $110 \mathrm{kV}$ lines in an oilfield substation are selected as the characteristic quantities of 
health index division, and the key characteristic quantities are weighted by entropy method to complete the distribution network health degree classification and calculation. Finally, through the generated health image and health index analysis, the fault warning and push operation and maintenance strategy are prompted.

\section{Availability of data and materials}

In this paper, the power factor, voltage deviation and line loss rate of seven lines of $110 \mathrm{KV}$ distribution network in an oilfield in a certain area are taken as the key characteristic quantity to calculate the network health index.The datasets generated and/or analysed during the current study are not publicly available due [REASON WHY DATA ARE NOT PUBLIC] but are available from the corresponding author on reasonable request.

\section{Funding}

The research was supported by Science and Development Foundation of Dong ying (DJ2020014) .The funding body did not influence the study at any stage (i.e., during design, data collection, analysis and interpretation of the data and writing the manuscript).

\section{Author information}

Affiliations

Yang Yang

Shengli College, School of Mechanical and Control Engineering, China University of Petroleum, Dongying Shandong China, 257061.

E-mail:000207@slcupc.edu.cn

Contributions

Yang Yang completed the data collection, calculation and writing of the paper.

Competing interests:

The authors declare that they have no competing interests.

\section{Abbreviations}

HI :Health Index;CBRM :Condition Based Risk Maintenance; PAS : Publicly Available Specification;AHP:analytic hierarchy process

\section{Declaration}




\section{Competing interests}

The authors declare that they have no confict of interest.

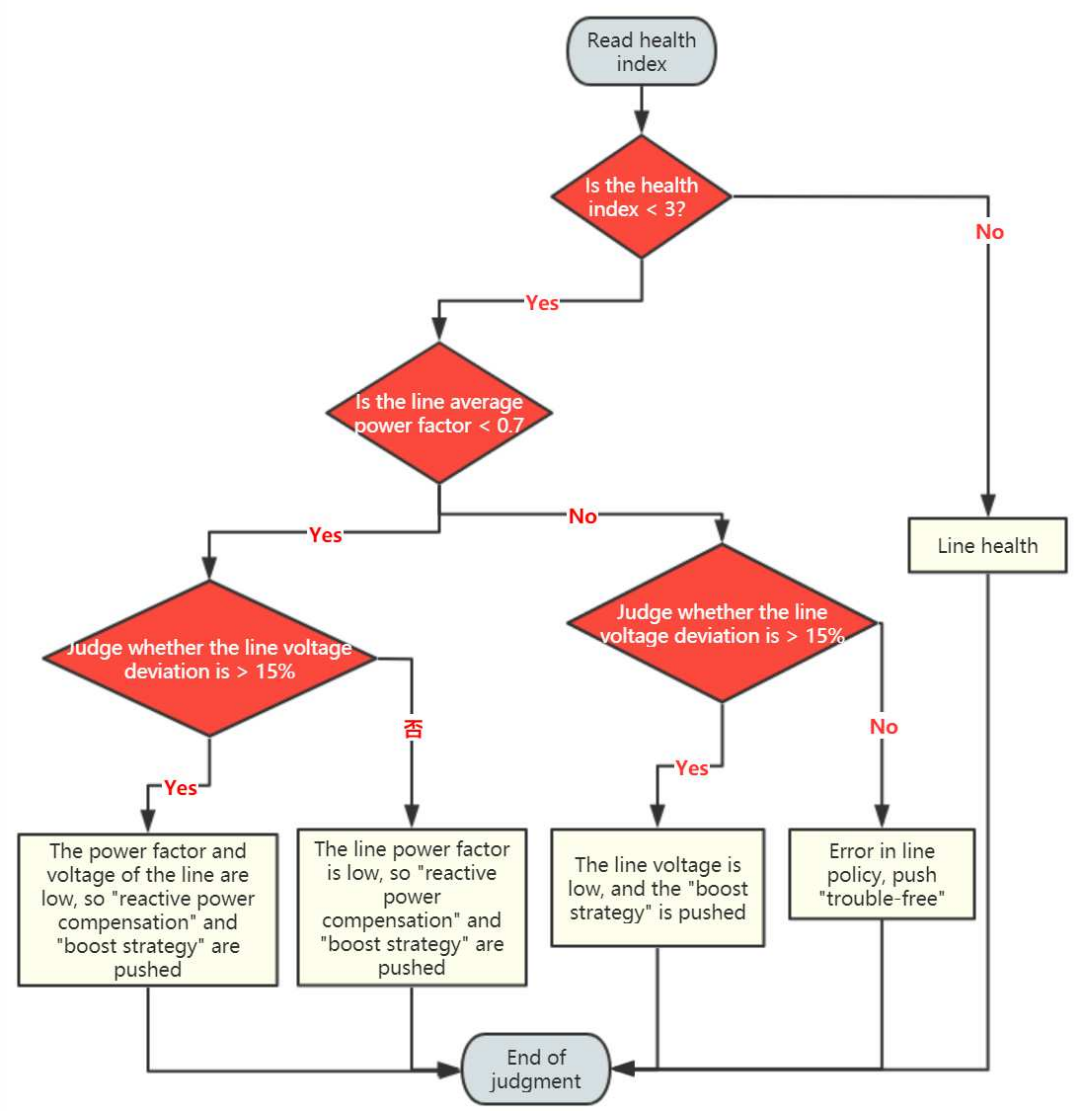

Fig1 The push process of operation and maintenance countermeasures

In an example, in order to better compare the changes of line loss health in one day, the line health portraits are displayed on the big data platform every day. It is stipulated that when the average health index of a certain line on that day is lower than 3 , the big data platform will issue a warning, that is, the health of a specific line shows general defects/serious defects/critical defects.

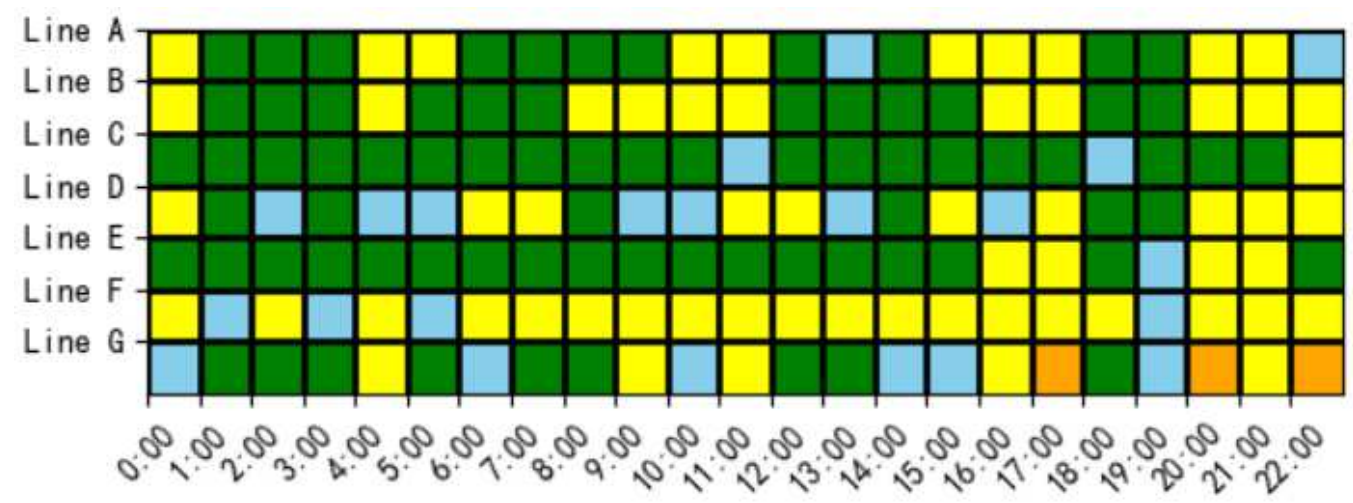

Fig2 Image of oilfield health on April 24th 
Results of health portrait on April 24, 2020 and operation and maintenance strategy.

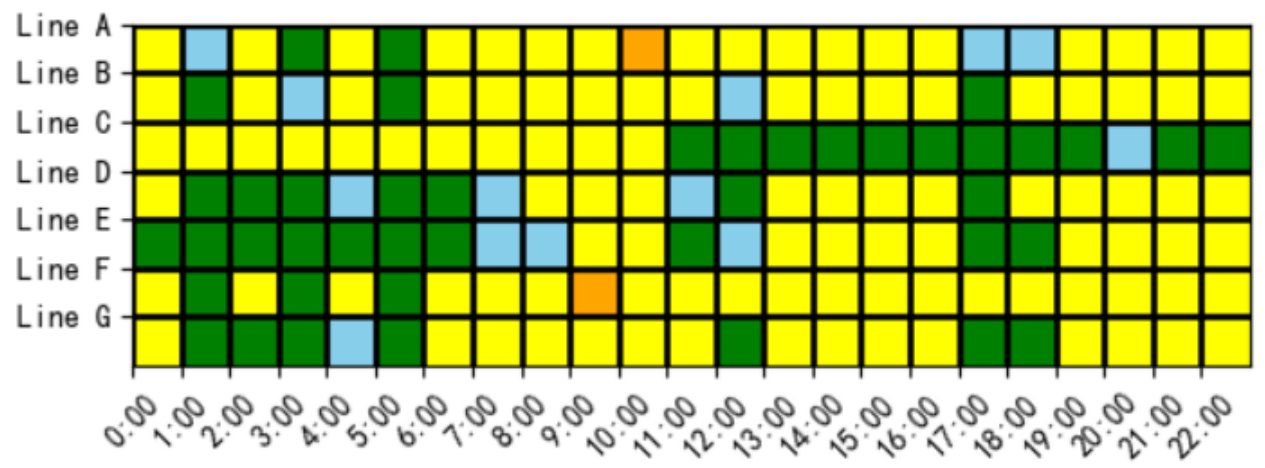

Fig.3 health degree portrait of oil field on May 2

Results of health portrait on May 2, 2020 and operation and maintenance strategy.

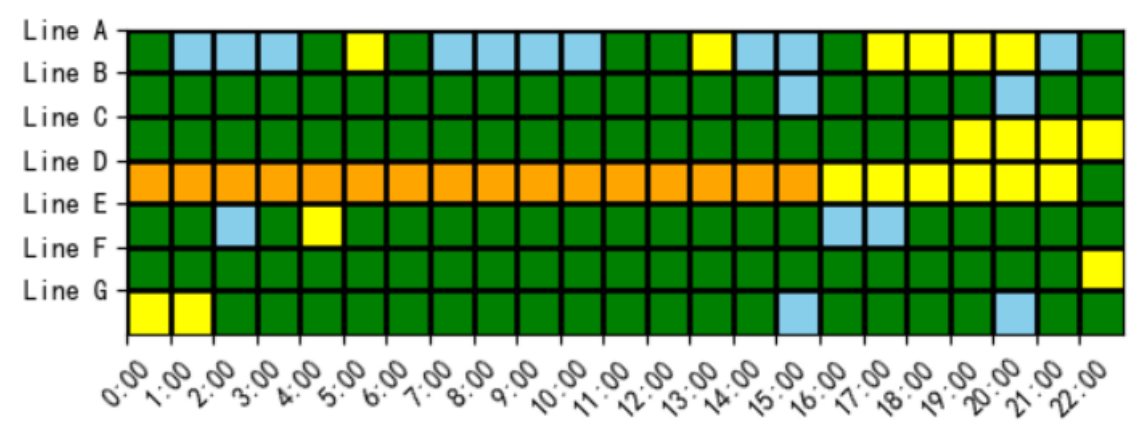

Fig.4 Health degree portrait of oil field on May 27

Results of health portrait on May 27, 2020 and operation and maintenance strategy.

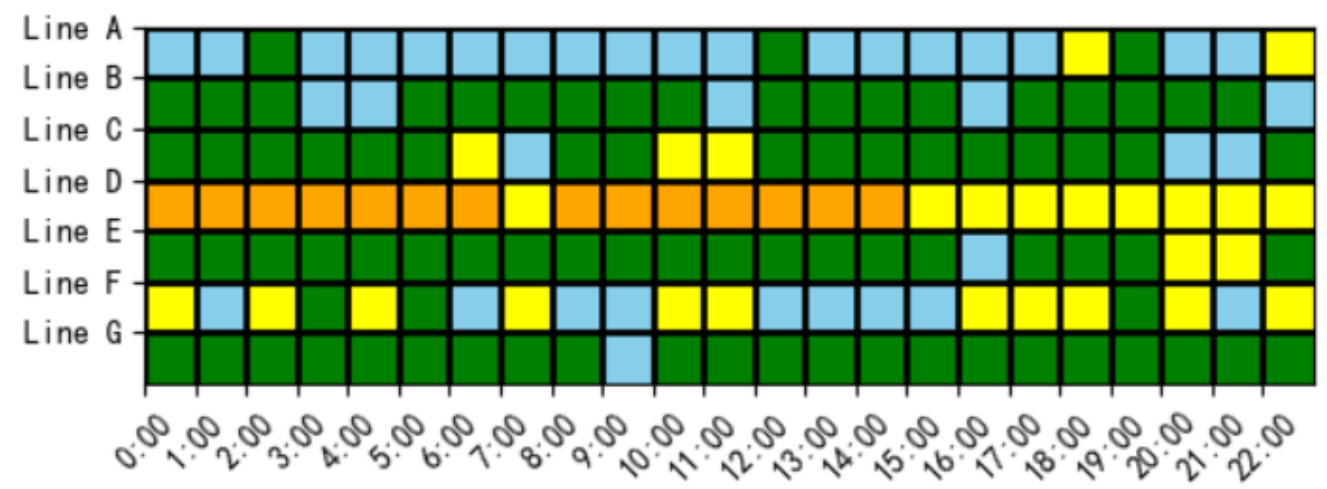

Fig. 5 health degree portrait of oil field on May 31

Results of health portrait on May 31, 2020 and operation and maintenance 
strategy. 